implicated in EMT. However, the accurate role of miR-361 in CC-associated EMT and the mechanisms underlying its function in CC remains largely unknown. The functional roles of miR-361 in CC cells were explored by a series of cell functional assays. Luciferase reporter assays were used to demonstrate the potential interaction between miR-361, HSP90 and long non-coding RNA (lncRNA) NEAT1. We detected a consistent reduction of miR-361 expression in CC tissues and CC cell lines, and miR-361 overexpression inhibited invasion and EMT phenotypes of CC cells by directly targeting a key EMT activator HSP90. Additionally, we detected significantly higher levels of HSP90 in CC tissues compared with normal tissues, and high expression of HSP90 predicted a poorer prognosis. We further identified NEAT1 as a significantly upregulated lncRNA in CC tissues and high expression of NEAT1 was associated with worse survival in CC patients. NEAT1 directly repressed miR-361 expression and played an oncogenic role in CC cell invasion and sphere formation. These results demonstrated that miR-361 directly targets HSP90 to inhibit the invasion and EMT features, and NEAT1 functions as an oncogenic lncRNA that suppresses miR-361 expression and induces EMT and sphere formation in CC cells, thus providing critical insights into the molecular pathways operating in this malignancy.

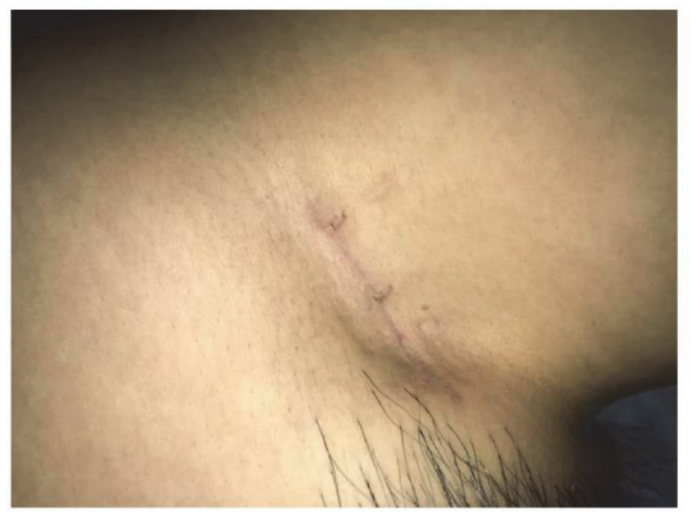

Appearance on the groin following M-MTIT

Abstract 321 Figure 1

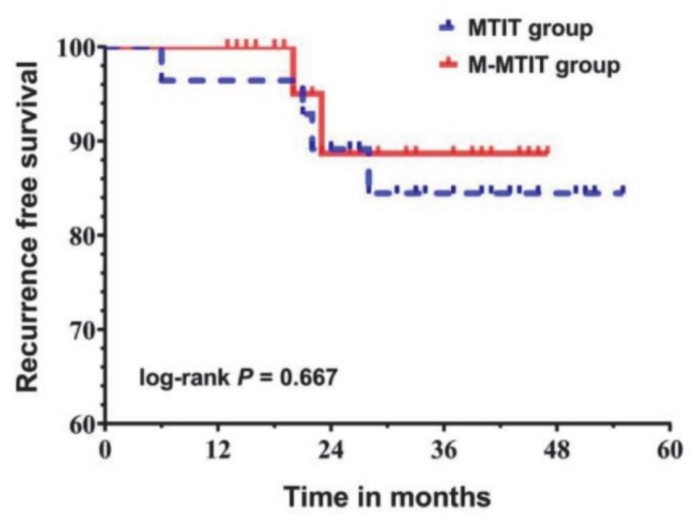

$2 \mathrm{~A}$
IGCS20_1342

\section{COMPARISON OF TWO TYPES OF TRIPLE INCISION TECHNIQUE IN THE TREATMENT OF PATIENTS WITH LOCALLY ADVANCED VULVAR CANCER}

${ }^{1} \mathrm{M}$ Wu, ${ }^{1} \mathrm{~J} \mathrm{Li},{ }^{2}{ }^{Y} \mathrm{Ma}^{*}$, ' ${ }^{1} \mathrm{Z}$ Lin. ${ }^{1}$ Sun Yat-sen Memorial Hospital, Sun Yat-sen University, China; ${ }^{2}$ Department of Obstetrics and Gynecology, the First Affiliated Hospital of Dalian Medical university, China

\subsection{6/ijgc-2020-IGCS.275}

Objective We proposed a modified triple incision technique (MTIT) for vulvar cancer patients with locally advanced disease and reported it in 2002. MTIT has gone through a series of modification and a modified MTIT (M-MTIT) came into being. The purpose of this study was to introduce M-MTIT and compare it with MTIT.

Methods 57 vulvar cancer patients with clinical stage T2 $(>4 \mathrm{~cm})$ or T3 disease were included. Of them, 28 underwent MTIT and 29 underwent M-MTIT. Their data on surgery-related complications and survival outcomes were compared.

Results The patients treated with M-MTIT developed significantly less surgery-related morbidities than patients treated

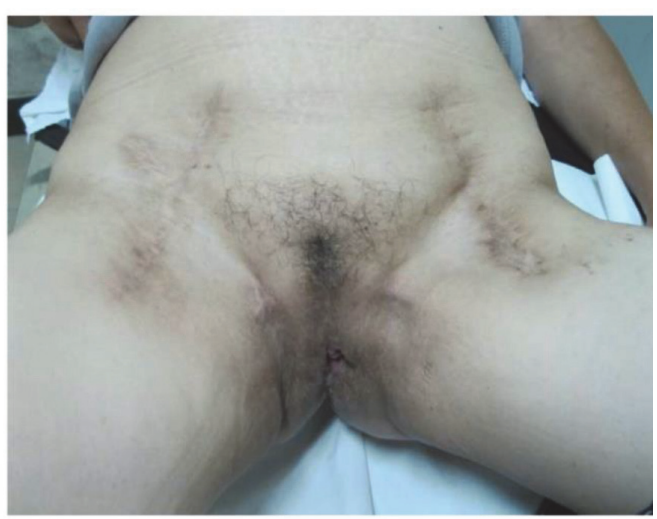

Appearance on the vulva and groin following $\mathrm{M}^{-\mathrm{MTI}^{-}}$

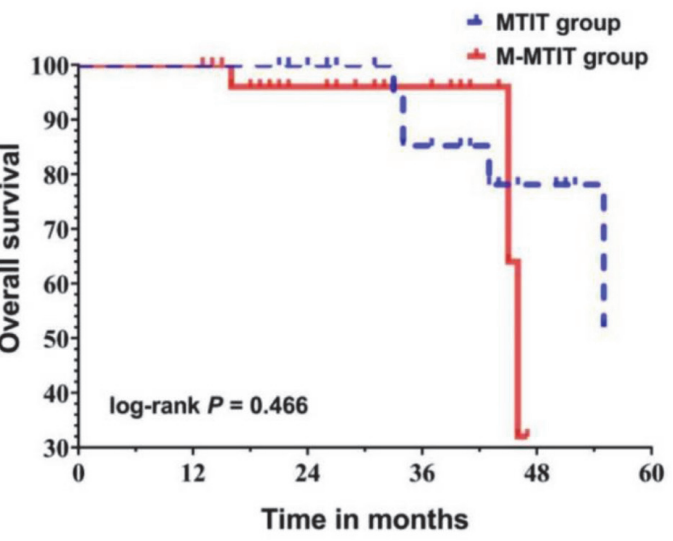

2B 
with MTIT (24.1\% vs. $60.7 \%, \mathrm{P}=0.005)$. Wound breakdown was the most common complication in our cohort, which occurred less frequently in the M-MTIT group than in the MTIT group $(10.3 \%$ vs. $35.7 \%, \mathrm{P}=0.022)$. Multivariate logistic regression analysis identified M-MTIT as an independent predictor of reduced risk of wound breakdown. The incidence of other complications, including lymphedema, wound infection and cellulitis was lower in M-MTIT group than in MTIT group; however, the differences did not reach statistical significance. Median followup time of this study was 33 months. The Kaplan-Meier survival graphs did not show significant differences in recurrence-free survival and overall survival between the two groups.

Conclusions M-MTIT correlates with lower morbidity rates and does not compromise oncological safety compared with MTIT. It could be considered as a safe and feasible option for vulvar cancer patients with locally advanced disease.

\section{IGCS20_1344}

\section{PREVALENCE OF HRHPV DNA AND P16/KI67 EXPRESSION AMONG WOMEN WITH CERVICAL DYSPLASIA}

VP*, A Sekar, R Nachiappa, R Dhodapkar. JIPMER, India

10.1136/ijgc-2020-IGCS.276

Objectives Markers such as HPV DNA, p16 and ki67 are helpful to decide who among the screen positives require further management and treatment. So, this study was planned to estimate the prevalence of HPV DNA, p16 and Ki67 expression among the women with cervical dysplasia and to correlate high risk HPV DNA positivity and P16/Ki67 expression among them.

Methods In this hospital-based cross-sectional study, 146 women with abnormal Pap smear reports were included in the study and were subjected to HPV DNA testing and colposcopy and directed biopsy for histopathology and immuno-histochemistry for p16 and ki67. Women who have already received treatment for dysplasia and women who were pregnant were excluded from the study.

Results Totally 146 women with abnormal Pap report with a mean age of 47.8 years were studied. The prevalence of highrisk HPV was $44.5 \%$ and HPV 16, 56 and 18 were the common genotypes. The prevalence of P16 and Ki67 expression more than $5 \%$ was $20.5 \%$ and $34.3 \%$ respectively. Positive correlation was noted between high risk HPV and P16/Ki67 expression ( $\mathrm{p}$ value of 0.0189 for P16 expression and HPV positivity, $p$ value of 0.0027 for Ki67 expression and HPV positivity).

Conclusions The prevalence of high-risk HPV in our study population comprising of women with abnormal Pap smears was $44.5 \%$. Positive correlation was noted between HPV, histopathology and P16 and Ki67 suggesting that these markers can be used as adjuncts in inconclusive cases during histopathological examination.
IGCS20_1346

323

ENGOT-EN9/LEAP-001: A PHASE 3 STUDY OF FIRST-LINE PEMBROLIZUMAB PLUS LENVATINIB COMPARED WITH CHEMOTHERAPY IN ADVANCED OR RECURRENT ENDOMETRIAL CANCER

${ }^{1} \mathrm{~L}$ Randall ${ }^{*},{ }^{2} \mathrm{C}$ Marth, ${ }^{3} \mathrm{C}$ Vulsteke, ${ }^{4} \mathrm{M}$ Jesús Rubio, ${ }^{5} \mathrm{~V}$ Makker, ${ }^{6} \mathrm{~B}$ Braicu, ${ }^{7} \mathrm{I} \mathrm{McNeish}$ ${ }^{8} \mathrm{R}$ Madry, ${ }^{9} \mathrm{~A}$ Ayhan, ${ }^{10} \mathrm{~K}$ Hasegawa, ${ }^{11} \mathrm{X} \mathrm{Wu},{ }^{12} \mathrm{~L}$ Dutta, ${ }^{13} \mathrm{C} \mathrm{Xu},{ }^{13} \mathrm{~S}$ Keefe, ${ }^{13} \mathrm{~J}$ Lee, ${ }^{14} S$ Pignata. ${ }^{1}$ Virginia Commonwealth University Medical Center, USA; ${ }^{2}$ Medical University of Innsbruck, Austria; ${ }^{3}$ AZ Maria Middelares, Belgium; ${ }^{4}$ Reina Sofía de Córdoba, Spain; ${ }^{5}$ Memorial Sloan Kettering Cancer Center, USA; ${ }^{6}$ Charité Universitätsmedizin, Germany; ${ }^{7}$ Imperial College, UK; ${ }^{8}$ Uniwersytet Medyczny im K. Marcinkowskiego w Poznaniu, Poland; ${ }^{9}$ Ankara Baskent University Hospital, Turkey; ${ }^{10}$ Saitama Medical University, Japan; ${ }^{11}$ Fudan University Shanghai Cancer Center, China; ${ }^{12}$ Eisai Inc., USA; ${ }^{13}$ Merck and Co., Inc., USA;

${ }^{14}$ National Cancer Institute, Italy

10.1136/ijgc-2020-IGCS.277

Background Prognosis and OS are poor in patients with advanced or recurrent endometrial cancer (EC). First-line standard of care for these patients is paclitaxel-carboplatin chemotherapy; however, more effective and tolerable therapies are needed. In the phase $1 \mathrm{~b} / 2$ trial KEYNOTE-146, which assessed the PD-1 inhibitor pembrolizumab combined with the multikinase inhibitor lenvatinib, an ORR of 38\% was observed in patients with previously treated advanced EC. ENGOT-en9/ LEAP-001 (NCT03884101) is a randomized, open-label, active-controlled, phase 3 study investigating pembrolizumab + lenvatinib vs chemotherapy in patients with EC.

Trial design Patients with newly diagnosed advanced (stage IIIIV) or recurrent EC not previously treated with antiangiogenic agents; systemic chemotherapy (unless within a chemoradiation regimen); PD-1, PD-L1, or PD-L2 inhibitors; or other T-cell receptor-targeted therapies will be eligible. Patients will be randomized $1: 1$ to receive pembrolizumab $200 \mathrm{mg}$ Q3W + lenvatinib $20 \mathrm{mg}$ daily or paclitaxel $175 \mathrm{mg} / \mathrm{m} 2 \mathrm{Q} 3 \mathrm{~W}+$ carboplatin AUC 6 Q3W. Randomization will be stratified by proficient vs deficient mismatch repair (pMMR vs dMMR) status. The pMMR population will be further stratified by prior chemoradiation (yes/no), measurable disease (yes/no), and ECOG performance status (0/1). Patients will receive treatment for $\leq 35$ cycles of pembrolizumab vs 7 cycles of chemotherapy or until initiation of a new anticancer treatment, unacceptable AEs, or withdrawal of consent. Primary endpoints are PFS (per RECIST v1.1 by blinded independent central review) and OS. Secondary endpoints are ORR, health-related QOL, safety/tolerability, and lenvatinib pharmacokinetics. Exploratory endpoints are disease control rate, clinical benefit rate, and duration of response. Enrollment is ongoing.

\section{IGCS20 1347}

\section{THE COMPLEXITY OF DECISION-MAKING FOR RISK- REDUCING SURGERY IN WOMEN WITH LYNCH SYNDROME}

H Cun ${ }^{*}$, D Nebgen, K Lu. MD Anderson Cancer Center, USA

\subsection{6/ijgc-2020-IGCS.278}

Introduction Risk-reducing surgery (RRS) in Lynch Syndrome effectively prevents endometrial and ovarian cancers. 